\title{
The Response of Extracellular Hydrogen Ion Concentration to Graded Degrees of Chronic Hypercapnia: The Physiologic Limits of the Defense of $\mathrm{pH}^{*}$
}

\author{
William B. Schwartz, Newton C. Brackett, Jr., $\dagger$ and \\ JORDAN J. COHEN $\dagger$ \\ (From the Department of Medicine, Tufts University School of Medicine, and the Renal \\ Laboratory, Pratt Clinic-New England Center Hospital, Boston, Mass.)
}

It is now well recognized that the defense of extracellular $\mathrm{pH}$ during acute hypercapnia depends upon generation of bicarbonate by body buffers (1). Recently, the quantitative characteristics of this defense have been defined in acute studies of the acid-base response of the intact organism to stepwise increases in carbon dioxide tension $(2,3)$. Similar observations are not available, however, for chronic hypercapnia in which defense of $\mathrm{pH}$ is enhanced by renal generation of bicarbonate.

The present study was undertaken to define the chronic adjustments of acid-base equilibrium in normal dogs subjected to stepwise elevations of arterial carbon dioxide tension to levels as high as $125 \mathrm{~mm} \mathrm{Hg}$. Observations have been carried out in an environmental chamber over a period of time sufficient to allow establishment of a steady state at each of three successive levels of carbon dioxide tension. The data demonstrate a highly reproducible bicarbonate response curve, the increment in extracellular bicarbonate concentration increasing notably in response to each stepwise elevation of $\mathrm{PCO}_{2}$. At no point was this increment sufficient to restore extracellular $\mathrm{pH}$ to normal; there was, however, a striking linearity in the relationship between hydrogen ion concentration and $\mathrm{PCO}_{2}$. These data indicate that extracellular acidity is defended with a virtually constant degree of effectiveness over the entire

* Submitted for publication August 7, 1964; accepted November 2, 1964.

Supported in part by grants from the National Heart Institute (H-759 and HTS 5309) and the Life Insurance Medical Research Fund.

$\dagger$ Work done during tenure of a postdoctoral research fellowship of the American Heart Association. range of carbon dioxide tensions that has been studied.

\section{Methods}

Balance studies were carried out in ten female mongrel dogs ranging in weight between 15.6 and $22.8 \mathrm{~kg}$. The animals were exposed chronically to successive increments in carbon dioxide tension utilizing an environmental chamber that has been previously described (4). The carbon dioxide concentration within the chamber can be maintained automatically within a range of approximately $\pm 0.5 \%$ of any desired level up to $20 \%$ and the oxygen concentration within $\pm 0.5 \%$ of any level between 10 and $30 \%$. A normal oxygen concentration of $21 \%$ was utilized throughout the present studies. The investigators entered the chamber through a transit lock, breathing through a full-face mask fitted with an appropriate air supply. The animals were kept in metabolic cages throughout the course of the study. The details of the balance technique have been previously described (5). Blood samples were drawn anaerobically into heparinized syringes from the femoral artery by percutaneous puncture.

The animals were fed $30 \mathrm{~g}$ per $\mathrm{kg}$ of an artificial diet, the composition of which has been described previously (5). The intrinsic electrolyte content of the diet was as follows: sodium, $1 \mathrm{mEq}$ per $100 \mathrm{~g}$; potassium, $0.1 \mathrm{mEq}$ per $100 \mathrm{~g}$; and chloride, $<1 \mathrm{mEq}$ per $100 \mathrm{~g}$. The diet was supplemented with $4 \mathrm{mEq}$ per $\mathrm{kg}$ of sodium and of chloride and $3 \mathrm{mEq}$ per $\mathrm{kg}$ of potassium as neutral phosphate $\left(4 \mathrm{HPO}_{4} / \mathrm{H}_{2} \mathrm{PO}_{4}\right)$. The diet was homogenized with 1.5 times its weight of water in the first four studies $(\operatorname{dogs} C, D, G$, and $H)$ and with twice its weight of water in all other studies.

Experimental design. Each study was initiated by a control period of 8 to 11 days; detailed balance observations were made over the last 5 to 8 control days and for the remainder of the study.

Previous observations indicated that 4 to 5 days of exposure were sufficient to establish a new steady state of acid-base equilibrium when a carbon dioxide atmosphere of $12 \%$ was employed $(5,6)$. For this reason the period of exposure at each level was never less than 5 days and in 
nearly every instance was 7 days or longer. Four levels of carbon dioxide $(7,11,15$, and $17 \%)$ were employed in the two initial studies, but only three levels $(7,11$, and $17 \%$ ) were employed in subsequent experiments, since it appeared that this latter protocol would define the response just as clearly and would be better tolerated by the animals.

Blood samples were obtained on 4 of the control days and, in most studies, on the first, third, fifth, and all subsequent days during each carbon dioxide period. The analytical procedures have been described in a previous paper from this laboratory (2). The daily balance was calculated as the net intake minus the combined outputs in urine and stool. The change in net external balance (or "delta balance") was calculated as the difference between the balance on an experimental day and the mean daily balance during the control period.

\section{General remarks}

\section{Results}

The ten dogs manifested remarkably few ill effects from exposure to carbon dioxide. They remained easy to manage and stood and walked normally, except in the $17 \%$ period, when slight ataxia regularly occurred and all required tube feeding. One dog (925) vomited shortly after exposure to $17 \%$ carbon dioxide, and two other dogs $(G$ and $H)$ died on day 2 of the $17 \%$ period because of an improper adjustment of the controls equipment that led to a lethal elevation of carbon dioxide tension; data from the final period in these three studies were, therefore, discarded. In one additional dog (929) a curious syndrome characterized by polyuria, hypernatremia, and weight loss occurred after a steady state had been established in the $17 \%$ period; observations during the polyuric phase were discarded.

Table I presents detailed data on an animal (932) who satisfactorily completed all experimental periods.

TABLE I

Balance data for a representative study illustrating the response

\begin{tabular}{|c|c|c|c|c|c|c|c|c|c|c|c|c|c|}
\hline & \multirow[b]{2}{*}{ Day } & \multirow[b]{2}{*}{$\begin{array}{c}\text { Body } \\
\text { wt. }\end{array}$} & \multicolumn{5}{|c|}{ Intake } & \multicolumn{6}{|c|}{ Urine } \\
\hline & & & $\mathrm{Na}$ & $\mathrm{Cl}$ & $\mathbf{K}$ & $\mathrm{N}$ & $\mathrm{H}_{2} \mathrm{O}$ & Vol & $\mathrm{pH}$ & $\mathrm{HCO}_{3}$ & $\mathrm{Cl}$ & $\mathrm{Na}$ & $\mathrm{K}$ \\
\hline & & $k g$ & \multicolumn{3}{|c|}{$m E q / d a y$} & $g / d a y$ & $m l / d a y$ & \multicolumn{3}{|l|}{$m l / d a y$} & \multicolumn{2}{|c|}{$m E q / d a y$} & \\
\hline \multirow[t]{6}{*}{ Control } & 1 & 22.9 & 90 & 90 & 59 & 12.2 & 1,630 & 1,200 & 6.17 & 6 & 80 & 72 & 55 \\
\hline & 2 & & 90 & 90 & 59 & 12.2 & 1,630 & 1,255 & 6.17 & 10 & 88 & 78 & 61 \\
\hline & 3 & & 89 & 89 & 59 & 12.1 & 1,610 & 1,120 & 6.25 & 9 & 69 & 57 & 54 \\
\hline & 4 & 22.8 & 90 & 90 & 59 & 12.2 & 1,630 & 1,390 & 6.19 & 8 & 87 & 81 & 63 \\
\hline & 5 & & 90 & 90 & 59 & 12.2 & 1,630 & 1,200 & 6.33 & 7 & 72 & 60 & 55 \\
\hline & 6 & 22.7 & 89 & 89 & 59 & 12.1 & 1,610 & $1,300 \dagger$ & & & 95 & 90 & 54 \\
\hline \multirow[t]{7}{*}{$7 \% \mathrm{CO}_{2}$} & 7 & 22.8 & 90 & 90 & 59 & 12.2 & 1,630 & 1,110 & 6.28 & 6 & 91 & 69 & 83 \\
\hline & 8 & & 90 & 90 & 59 & 12.2 & 1,630 & 1,170 & 6.24 & 6 & 70 & 52 & 52 \\
\hline & 9 & 22.8 & 89 & 89 & 59 & 12.1 & 1,610 & $1 ; 660$ & 6.50 & 25 & 119 & 125 & 71 \\
\hline & 10 & & 89 & 89 & 59 & 12.1 & 1,610 & 1,060 & 5.96 & 3 & 64 & 43 & 46 \\
\hline & 11 & & 89 & 89 & 59 & 12.1 & 1,610 & 1,420 & 6.46 & 15 & 92 & 101 & 61 \\
\hline & 12 & & 90 & 90 & 59 & 12.2 & 1,630 & 950 & 6.40 & 7 & 47 & 44 & 36 \\
\hline & 13 & & 88 & 88 & 58 & 11.9 & 1,600 & 1,470 & 6.08 & 9 & 109 & 96 & 71 \\
\hline \multirow[t]{7}{*}{$11 \% \mathrm{CO}_{2}$} & 14 & 23.0 & 90 & 90 & 59 & 12.2 & 1,630 & 960 & 6.69 & 31 & 67 & 86 & 99 \\
\hline & 15 & & 89 & 89 & 59 & 12.1 & 1,610 & 1,100 & 6.38 & 12 & 103 & 73 & 63 \\
\hline & 16 & & 90 & 90 & 59 & 12.2 & 1,630 & 1,210 & 6.56 & 24 & 110 & 99 & 51 \\
\hline & 17 & 23.3 & 89 & 89 & 59 & 12.1 & 1,610 & 700 & 6.52 & 9 & 44 & 52 & 36 \\
\hline & 18 & 23.2 & 90 & 90 & 59 & 12.2 & 1,630 & 1,000 & 6.52 & 20 & 111 & 98 & 72 \\
\hline & 19 & 23.2 & 89 & 89 & 59 & 12.1 & 1,610 & 1,000 & 6.74 & 30 & 62 & 75 & 50 \\
\hline & 20 & & 89 & 89 & 59 & 12.1 & 1,610 & 970 & 6.58 & 23 & 100 & 87 & 59 \\
\hline \multirow[t]{11}{*}{$17 \% \mathrm{CO}_{2}$} & 21 & 23.1 & 88 & 88 & 58 & 11.9 & 1,600 & 510 & 6.83 & 37 & 43 & 101 & 79 \\
\hline & 22 & & 88 & 88 & 59 & 12.1 & 1,610 & 510 & 6.36 & 10 & 87 & 57 & 84 \\
\hline & 23 & 23.2 & 88 & 88 & 59 & 12.1 & 1,610 & 670 & 6.65 & 23 & 103 & 91 & 58 \\
\hline & 24 & 22.7 & 90 & 90 & 60 & 12.3 & 1,650 & $1,130 \dagger$ & & & 138 & 96 & 66 \\
\hline & 25 & & 88 & 88 & 59 & 12.1 & 1,610 & 795 & 6.66 & 29 & 66 & 82 & 46 \\
\hline & 26 & 22.7 & 90 & 90 & 60 & 12.3 & 1,650 & 910 & 6.49 & 17 & 117 & 77 & 54 \\
\hline & 27 & 22.7 & 88 & 88 & 59 & 12.1 & 1,610 & 740 & 6.65 & 25 & 66 & 66 & 50 \\
\hline & 28 & 22.5 & 88 & 88 & 59 & 12.1 & 1,610 & 880 & 6.70 & 33 & 100 & 100 & 60 \\
\hline & 29 & & 87 & 87 & 58 & 11.9 & 1,600 & 595 & 6.67 & 23 & 61 & 60 & 52 \\
\hline & 30 & 22.5 & 88 & 88 & 59 & 12.1 & 1,610 & 940 & 6.75 & 36 & 98 & 89 & 61 \\
\hline & 31 & 22.4 & 89 & 89 & 59 & 12.2 & 1,630 & 950 & 6.92 & 40 & 90 & 88 & 57 \\
\hline
\end{tabular}

* TA = titratable acid

† Urine contaminated with fecal material. 
Plasma changes

1) Acid-base. a) Evidence that a chronic steady state was achieved at each level of carbon dioxide. Figure 1 presents the day-to-day changes in the acid-base parameters of the plasma for two representative studies. Figure 1 shows that each change in inspired carbon dioxide initiates the following events: 1) arterial $\mathrm{PcO}_{2}$ rises abruptly and then remains virtually constant, 2) plasma bicarbonate concentration rises over the first several days and appears to reach a new steady state between the third and fifth days, and as a result 3) plasma hydrogen ion concentration apparently reaches a new steady state between 3 and 5 days. Statistical analysis of observations from all experiments demonstrated no trend in arterial $\mathrm{PCO}_{2}$, bicarbonate, or hydrogen ion concentrations over the last 3 days of each period; the average change in bicarbonate concentration over this interval was $+0.1 \mathrm{mEq}$ per $\mathrm{L}$ or less at each level of exposure to carbon dioxide.

To examine the possibility that bicarbonate concentration might have changed during these last 3 days at a rate too slow to be detectable under the conditions of the present study, three additional long-term studies were done. In these studies dogs were maintained in an uninterrupted $10 \%$ carbon dioxide environment for 6 weeks. The results of these studies (Figure 2) also indicated that no further adjustments of acid-base equilibrium occurred beyond the fifth day of exposure to high carbon dioxide.

b) Plasma acid-base parameters during the steady state. In Figure 3 the steady-state bicarbonate $/ \mathrm{PCO}_{2}$ relationships are shown for all ten studies. The chronic steady-state response of each animal to a given level of carbon dioxide is represented by a single point obtained by

TABLE I

to three chronically sustained levels of carbon dioxide (dog 932)

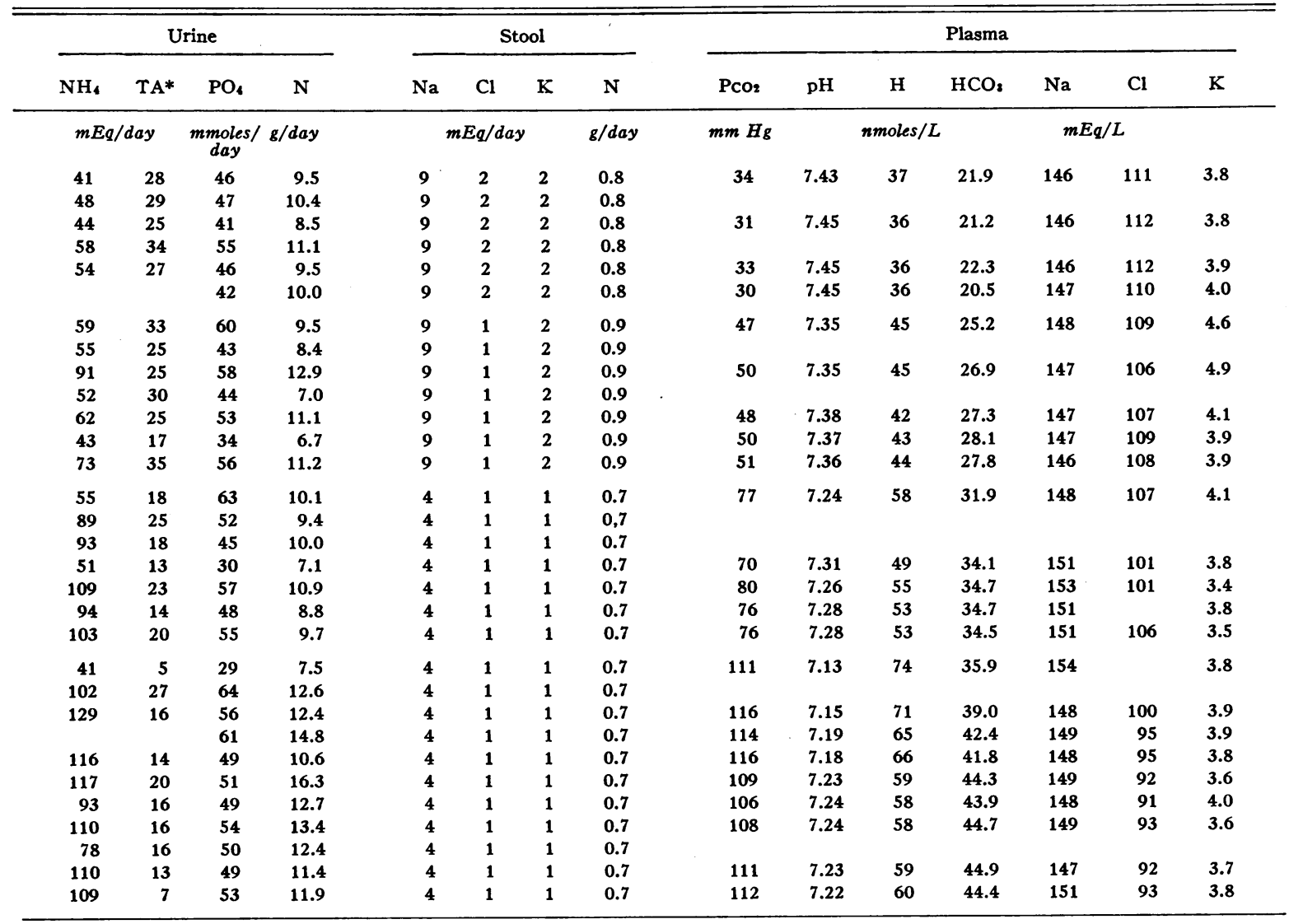




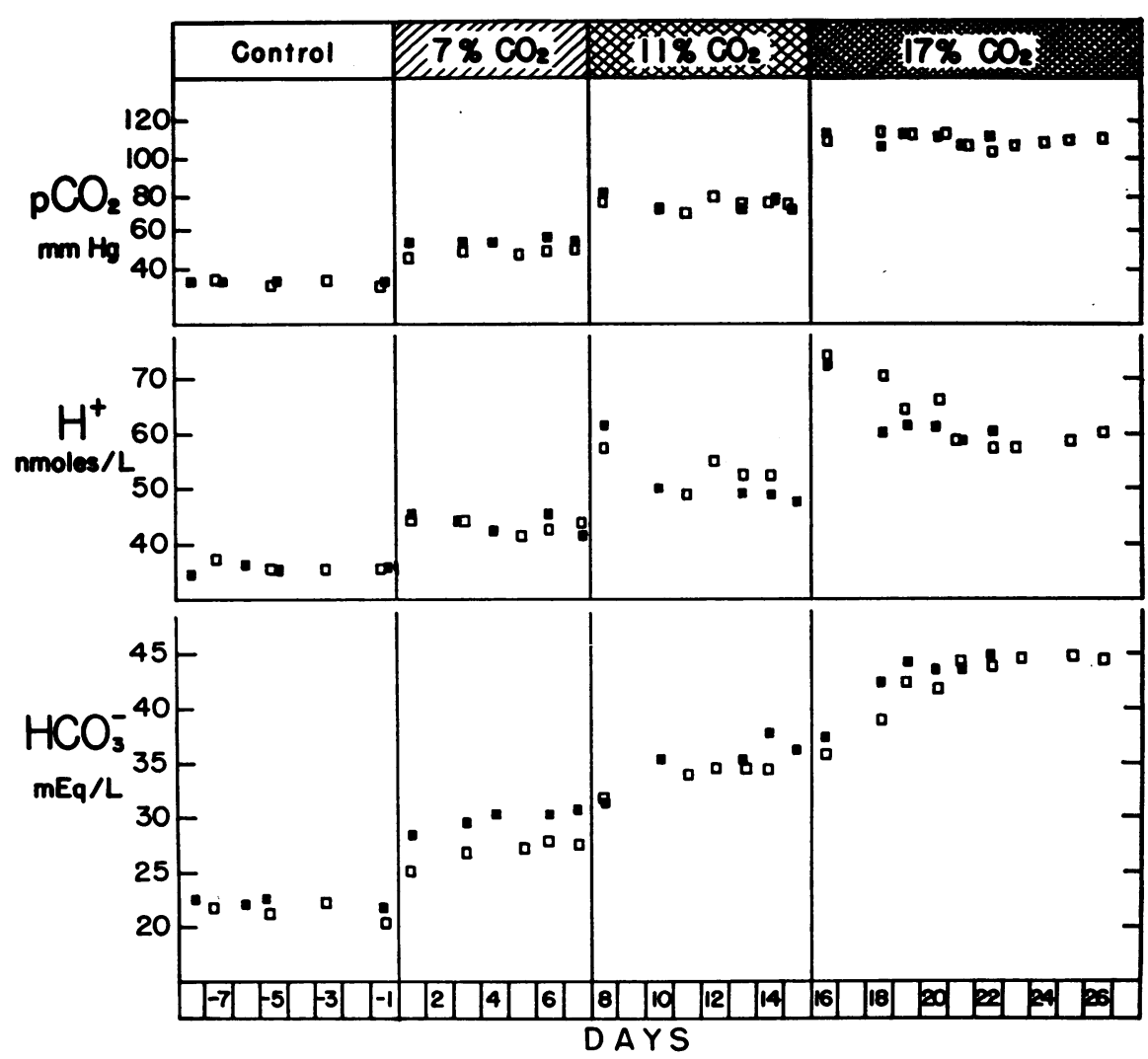

Fig. 1. Plasma hydrogen ion concentration, bicarbonate concentration, and $\mathrm{PCO}_{2}$ DURING THE TIME COURSE OF TWO REPRESENTATIVE STUdIES (DOGS 932 AND 944). Each symbol represents a single arterial blood sample obtained on the day indicated on the abscissa. Note that a steady state was apparently achieved 3 to 5 days after exposure to each new level of carbon dioxide.

averaging the observations made on the last 3 days of each period. Note that there was a marked rise in plasma bicarbonate concentration with each elevation of inspired $\mathrm{PCO}_{2}$, the increment in bicarbonate tending to diminish slightly at the higher $\mathrm{PCO}_{2}$ levels. The dotted line, shown for comparison, represents the bicarbonate $/ \mathrm{PCO}_{2}$ relationship observed during earlier studies of acute hypercapnia (2).

In Figure 4 the steady-state hydrogen ion concentrations are related to the arterial $\mathrm{PCO}_{2}$ values. Hydrogen ion concentration rose in response to each increase in carbon dioxide tension, $\mathrm{pH}$ never being returned to control levels. Furthermore, the increment in hydrogen ion concentration appears to be proportionate to the degree of elevation of $\mathrm{PCO}_{2}$. This linear relationship was observed in all ten studies; the individual regression lines ranged in slope between 0.23 and 0.40 and in intercept between 21.9 and 32.2. The correlation coefficients averaged 0.97 (range, 0.92 to 0.99$)$. The slope (0.32) and intercept (26.9) of the solid line drawn through the points in Figure 4 were obtained by averaging the slopes and intercepts of the individual regressions (see Appendix). The dotted line shown for comparison represents the average hydrogen ion $/ \mathrm{PCO}_{2}$ relationship observed during previous studies of acute hypercapnia (2).

The linear hydrogen ion $/ \mathrm{PCO}_{2}$ relationship observed in the present study has provided a basis for the calculation of a significance band that defines, with an estimated $95 \%$ probability, the change in extracellular acidity to be anticipated during uncomplicated chronic hypercapnia (see Appendix). An equivalent band for bicarbonate was then obtained by appropriate substitutions in the Henderson-Hasselbalch equation. The 
limits of these bands over the range of carbon dioxide tensions that has been studied are shown in Figure 5 and Table II.

2) Plasma electrolytes. Plasma chloride concentration fell significantly with each increase in inspired carbon dioxide $(p<0.01)$, averaging $113 \mathrm{mEq}$ per $\mathrm{L}$ during the control period and 95 $\mathrm{mEq}$ per $\mathrm{L}$ during the $17 \%$ carbon dioxide period. Sodium concentration increased from $146 \mathrm{mEq}$ per L during control to $150 \mathrm{mEq}$ per L $(p<0.02)$ during the $17 \%$ carbon dioxide period. Plasma potassium, phosphate, and unmeasured anion concentrations did not change significantly.

\section{Balance data}

Balance data are presented in Tables III and IV for all eight animals subjected to the standard protocol of exposure to 7,11 , and $17 \%$ carbon dioxide.
1) Acid-base (Figure 6 and Table III). The qualitative changes in the acid-base parameters of the urine in response to each level of carbon dioxide were similar to those described previously in studies of prolonged exposure to $12 \%$ carbon dioxide (5). A feature common to all levels of exposure was a marked augmentation in net acid excretion which, when accumulated by period, averaged $77 \mathrm{mEq}$ during the $7 \%$ exposure, 126 $\mathrm{mEq}$ during the $11 \%$ exposure, and $171 \mathrm{mEq}$ during the $17 \%$ exposure. These increases resulted from a large increment in ammonium excretion that more than offset a simultaneous suppression of titratable acid and increase in bicarbonate excretion. Daily bicarbonate excretion exceeded the control values during all periods and averaged about 15 to $20 \mathrm{mEq}$ per day during the $17 \%$ carbon dioxide period; the factors responsible for this persistent bicarbonate diuresis are obscure.

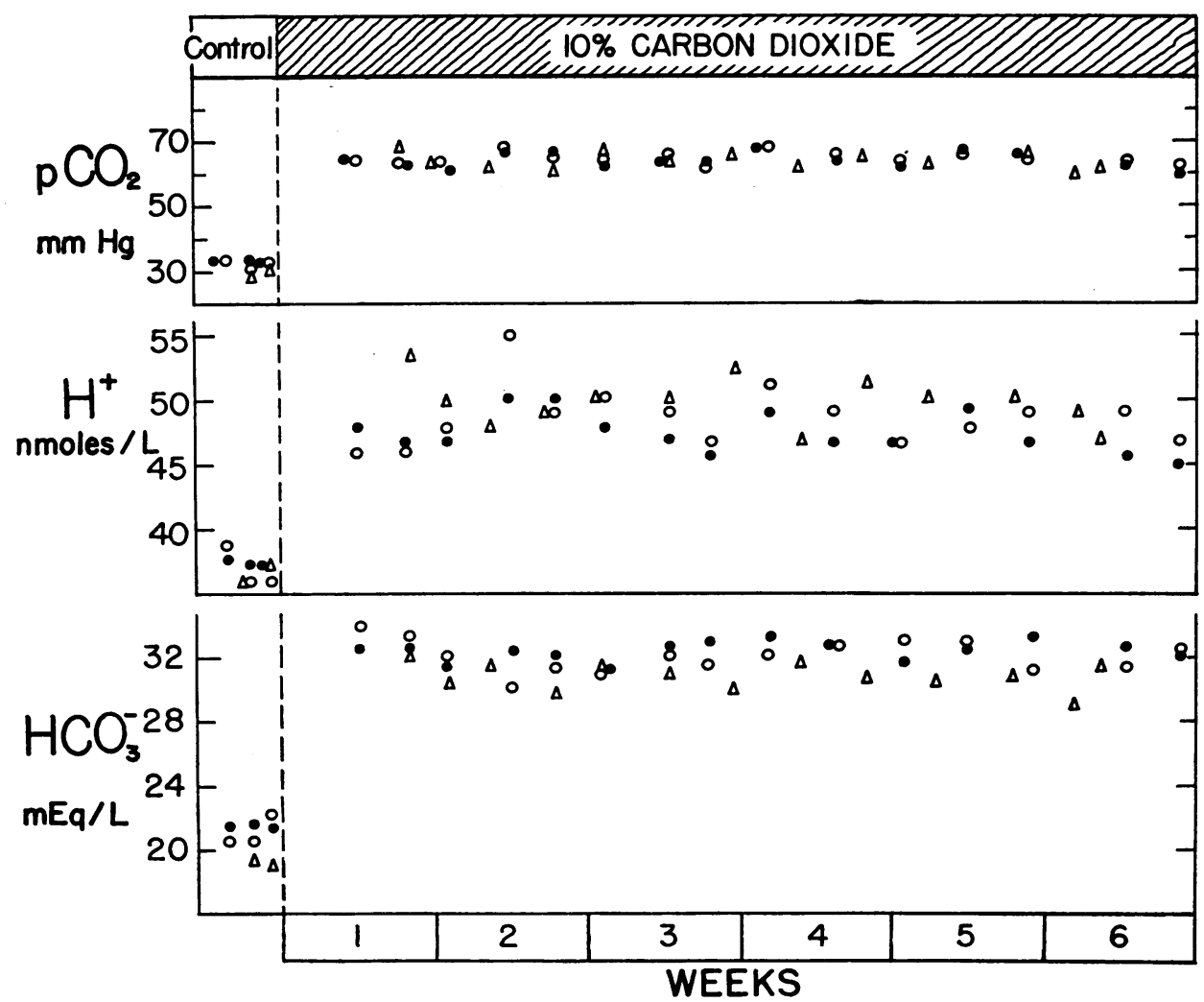

Fig. 2. Plasma hydrogen ion concentration, bicarbonate concentration, and Pco 2 FOR THREE ANIMALS EXPOSED TO A $10 \%$ CARBON DIOXIDE ENVIRONMENT FOR A 6-WEEK PERIOD. Note that a steady state was achieved within 5 days after exposure of the animals to carbon dioxide. 


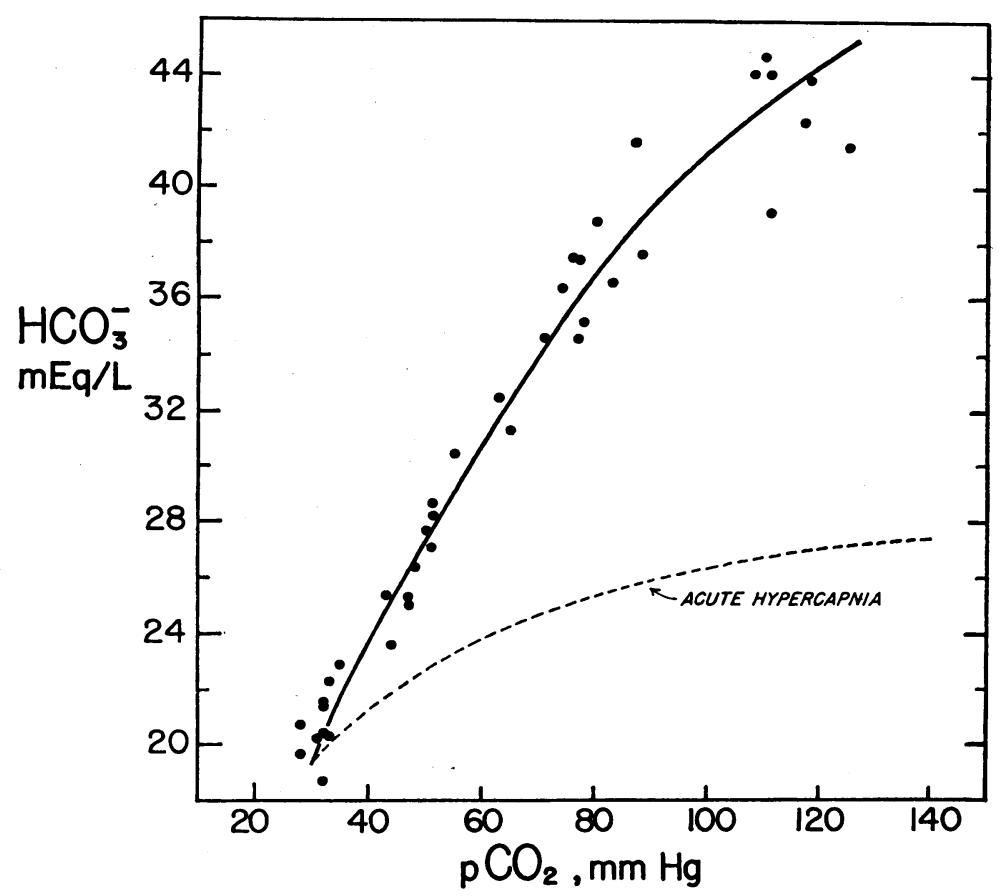

Fig. 3. Steady-State Relationship betweEN plasma bicarbonate conCENTRATON AND $\mathrm{PCO}_{2}$ DURING CHRONIC HYPERCAPNIA OF INCREASING SEVERITY. Steady-state bicarbonate values for all ten studies are shown. Each point represents the average of the plasma bicarbonate concentrations observed on the last 3 days at each level of exposure. The dotted line represents the mean bicarbonate response curve previously observed in the dog during acute hypercapnia. The curve relating bicarbonate and $\mathrm{PCO}_{2}$ was drawn by inspection.

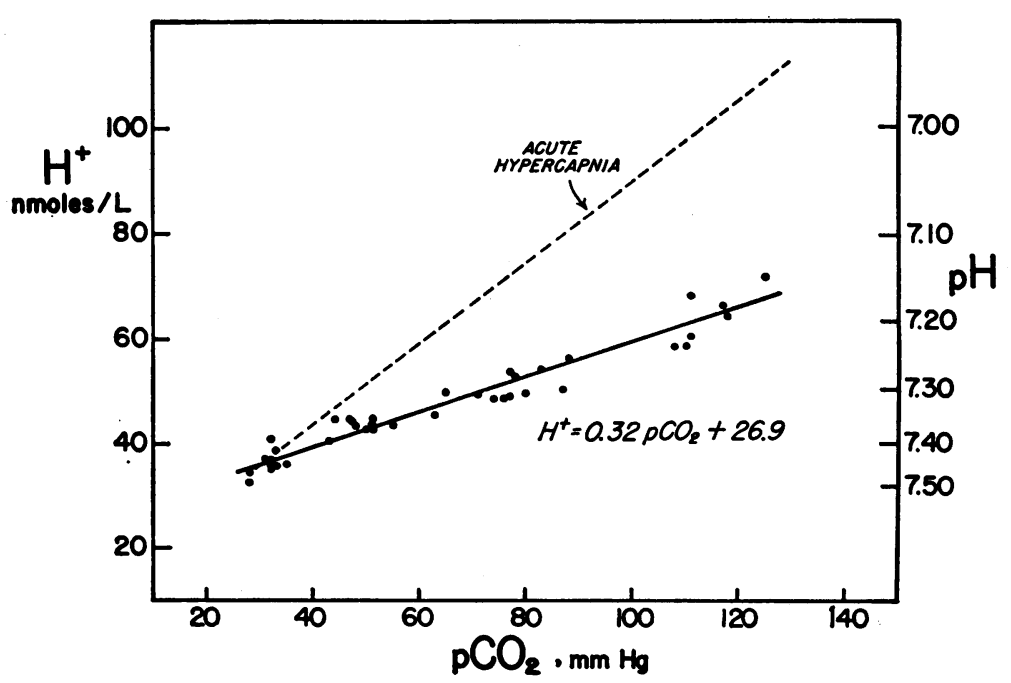

Fig. 4. STEADY-STAte RELATIONSHIP BETWEEN HYDROGEN ION CONCENTRATION AND $\mathrm{PCO}_{2}$ DURING CHRONIC HYPERCAPNIA OF INCREASING SEVERITY. Steady-state hydrogen ion values are shown for all ten studies. Each point represents the average of the plasma hydrogen ion concentrations observed on the last 3 days at each level of exposure. The line drawn through the points was obtained by averaging the slopes and intercepts of the individual regression lines (see Appendix). 


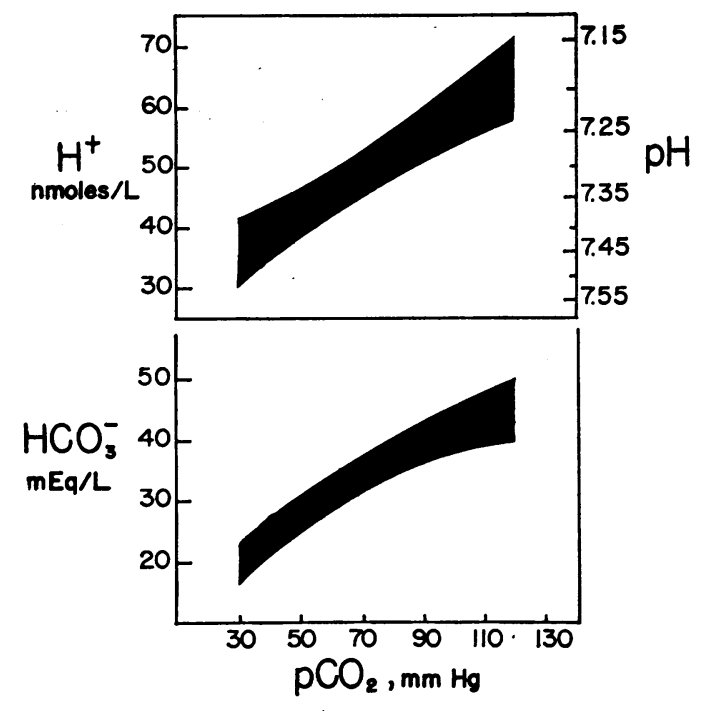

Fig. 5. Significance BANDS FOR PLASMA HYDROGEN ION AND BICARBONATE CONCENTRATIONS IN CHRONIC HYPERCAPNIA. The significance band for hydrogen ion is shown in the upper portion of the Figure and the equivalent significance band for bicarbonate in the lower portion. In uncomplicated chronic hypercapnia values for hydrogen ion and bicarbonate concentrations will, with an estimated $95 \%$ probability, fall within the respective zones. Observations lying outside these bands would indicate the presence of a complicating metabolic acidbase disturbance.

Figure 6 underscores a striking difference between the urine composition on the first day of the $11 \%$ and $17 \%$ periods as compared to subsequent days. Bicarbonate excretion was appreciably higher on this day, whereas both ammonium and titratable acid excretion were suppressed; as a result net acid excretion fell considerably below control levels. By contrast, in the 7\% period there was no significant bicarbonate diuresis on the first day of exposure, and net acid excretion remained at control values or rose slightly. These findings suggest that the renal threshold for bicarbonate was exceeded by the generation of bicarbonate from endogenous buffer stores on the first day of the 11 and $17 \%$ periods but not on the first day of the $7 \%$ period.

2) Electrolyte, nitrogen, and internal balances (Table IV). Sodium balance was negative in nearly every instance during the first two experimental periods (average, $-41 \mathrm{mEq}$ at $7 \%$ carbon dioxide and $-36 \mathrm{mEq}$ at $11 \%$ carbon dioxide), but showed no clear trend during the
TABLE II

Significance bands for plasma hydrogen ion and bicarbonate concentrations during chronic hypercapnia in the dog*

\begin{tabular}{|c|c|c|c|c|c|c|}
\hline \multirow{2}{*}{$\frac{\mathrm{PcO} 2}{m m \mathrm{Hg}}$} & \multicolumn{4}{|c|}{$\mathrm{H}$} & \multicolumn{2}{|c|}{$\mathrm{HCO}_{3}$} \\
\hline & \multicolumn{2}{|c|}{ nmoles $/ L$} & \multicolumn{2}{|c|}{$p H$} & \multicolumn{2}{|c|}{$m E q / L$} \\
\hline 30 & 31.2 & 41.6 & 7.51 & 7.38 & 17.2 & 23.0 \\
\hline 40 & 35.0 & 44.0 & 7.46 & 7.36 & 21.7 & 27.3 \\
\hline 50 & 38.8 & 46.6 & 7.41 & 7.33 & 25.6 & 30.8 \\
\hline 60 & 42.1 & 49.5 & 7.38 & 7.31 & 29.0 & 34.1 \\
\hline 70 & 45.3 & 52.7 & 7.34 & 7.28 & 31.7 & 36.9 \\
\hline 80 & 48.0 & 56.2 & 7.32 & 7.25 & 34.0 & 39.8 \\
\hline 90 & 50.6 & 60.0 & 7.30 & 7.22 & 35.9 & 42.5 \\
\hline 100 & 53.0 & 63.8 & 7.28 & 7.20 & 37.5 & 45.1 \\
\hline 110 & 55.3 & 67.9 & 7.26 & 7.17 & 38.7 & 47.5 \\
\hline 120 & 57.5 & 71.9 & 7.24 & 7.14 & 39.9 & 49.9 \\
\hline
\end{tabular}

* These limits contain, with an estimated $95 \%$ probability, the anticipated responses during uncomplicated chronic hypercapnia.

$17 \%$ period. Chloride balance followed a pattern similar to that for sodium. "Selective" chloride balance, calculated by using the ratio of sodium to chloride concentrations in the extracellular fluid (arbitrarily taken as 1.3 ), averaged $-21 \mathrm{mEq}$ during the $7 \%$ period, $-40 \mathrm{mEq}$ during the $11 \%$ period, and $-25 \mathrm{mEq}$ during the $17 \%$ period.

\section{TABLE III}

Changes in titratable acid, ammonium, bicarbonate, and net acid excretion during chronic exposure to three levels of carbon dioxide*

\begin{tabular}{|c|c|c|c|c|c|c|}
\hline Period & Dog & Days & TA & $\mathrm{NH}_{4}$ & $\mathrm{HCO}_{2}$ & $\begin{array}{l}\text { Net } \\
\text { acid }\end{array}$ \\
\hline & & & $m E q$ & $m E q$ & $m E q$ & $m E q$ \\
\hline \multirow[t]{9}{*}{$7 \% \mathrm{CO}_{2}$} & G & 7 & +32 & +52 & -5 & +89 \\
\hline & $\mathbf{H}$ & 7 & -17 & +149 & +16 & +116 \\
\hline & 925 & 7 & -4 & +178 & +22 & +152 \\
\hline & 926 & 7 & -19 & +47 & +14 & +14 \\
\hline & 929 & 7 & -21 & +55 & +5 & +29 \\
\hline & 932 & 7 & -13 & +92 & +15 & +64 \\
\hline & 942 & 7 & -15 & +107 & +14 & +78 \\
\hline & 944 & 7 & -34 & +141 & +32 & +75 \\
\hline & \multicolumn{2}{|c|}{ Average } & -11 & +103 & +14 & +77 \\
\hline \multirow[t]{9}{*}{$11 \% \mathrm{CO}_{2}$} & G & 9 & -31 & +201 & +24 & +146 \\
\hline & $\mathrm{H}$ & 9 & -41 & +321 & +59 & +221 \\
\hline & 925 & 9 & -36 & +318 & +89 & +193 \\
\hline & 926 & 9 & -112 & +175 & +51 & +12 \\
\hline & 929 & 7 & -76 & +203 & +57 & +70 \\
\hline & 932 & 7 & -72 & +251 & +93 & +86 \\
\hline & 942 & 8 & -52 & +230 & +56 & +122 \\
\hline & 944 & 8 & -84 & +339 & +95 & +160 \\
\hline & \multicolumn{2}{|c|}{ Average } & -63 & +255 & +66 & +126 \\
\hline \multirow[t]{6}{*}{$17 \% \mathrm{CO}_{2}$} & 926 & 6 & -76 & +205 & +43 & +86 \\
\hline & 929 & 6 & -96 & +182 & +61 & +25 \\
\hline & 932 & 11 & -154 & +588 & +211 & +223 \\
\hline & 942 & 9 & -81 & +597 & +142 & +374 \\
\hline & 944 & 7 & -57 & +277 & +75 & +145 \\
\hline & \multicolumn{2}{|c|}{ Average } & -93 & +370 & +106 & +171 \\
\hline
\end{tabular}

* Data for each period have been accumulated separately. 
Nitrogen balance tended to be negative in all periods, but was uniformly so only in the 11 and $17 \%$ periods.

Potassium excretion increased above control levels on the first day of each period with a resultant delta balance for this day amounting to -30 to $-50 \mathrm{mEq}$. Potassium was subsequently retained during each period, and as a consequence, potassium balance was virtually restored to normal at the end of the $7 \%$ period and was only slightly negative at the end of the 11 and $17 \%$ periods. These remaining potassium deficits could be completely accounted for by the concomitant negative nitrogen balances, indicating that a persistent, "selective" potassium depletion did not occur.

Phosphorus excretion followed a pattern similar to that for potassium, increasing about 20 mmoles above the average control level on the day following each increase in carbon dioxide concentration. Phosphate excretion tended to return towards control levels subsequently, but remained slightly elevated during each period (about 5 mmoles per day). As is well recognized (2), there is a rise in both plasma potassium and phosphorus concentrations in response to acute elevations of carbon dioxide tension, a finding which suggests that the initial kaluresis and phosphaturia noted here resulted from increased loads rather than from a primary reduction of renal threshold for these substances.

No appreciable shifts of sodium or potassium occurred between intra- and extracellular compartments (Table IV) during any of the three experimental periods. Extracellular fuid volume, as estimated from "chloride space" calculations, remained virtually unchanged throughout the study.

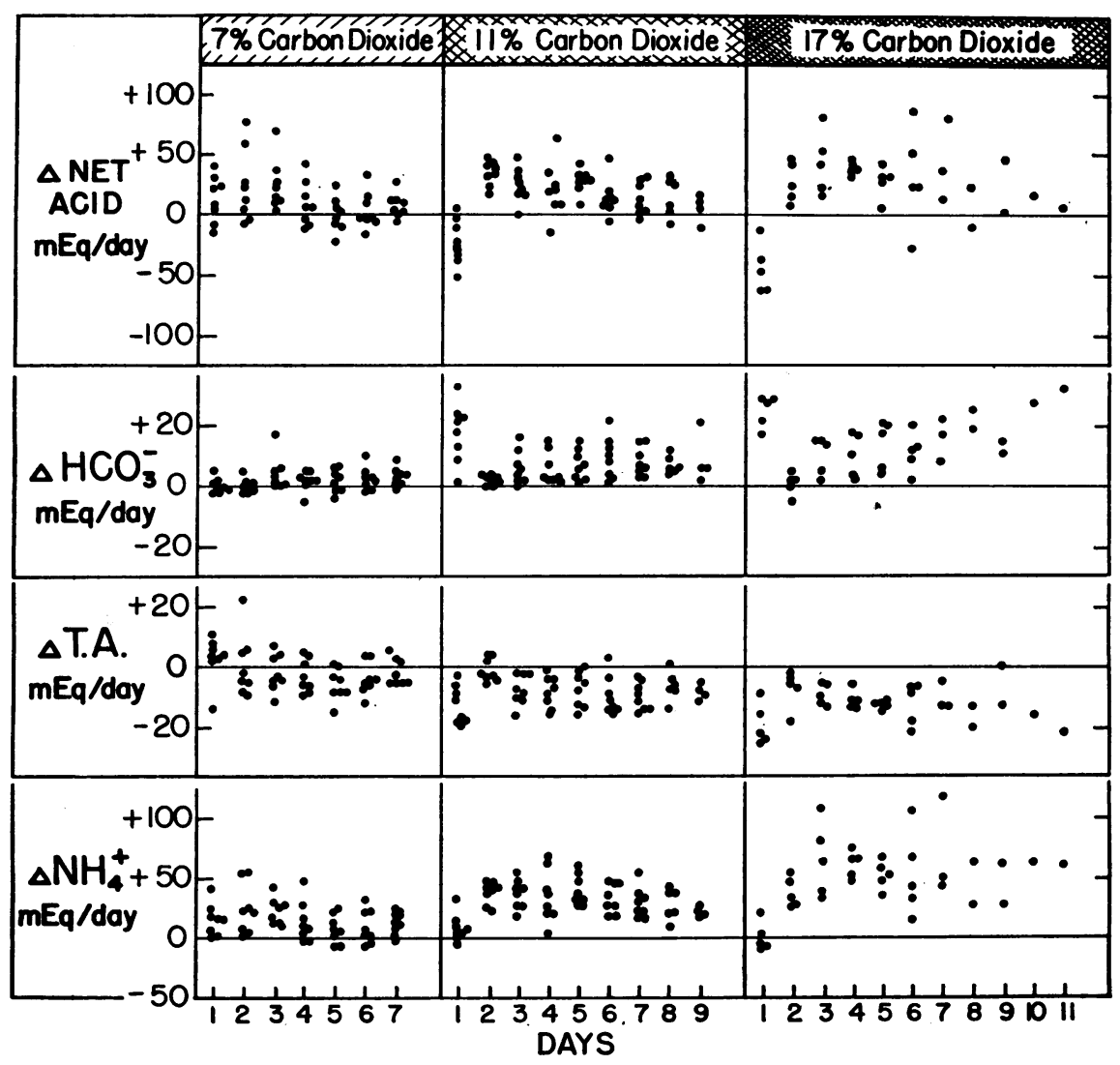

Fig. 6. Change in the excretion of ammonium, titratable acid, and bicarbonate DURING EXPOSURE TO THREE LEVELS OF CARBON DIOXIDE. The values represent changes from the mean daily excretion during the control period. 
RESPONSE TO GRADED DEGREES OF CHRONIC HYPERCAPNIA

TABLE IV

Change in balances during chronic exposure to three levels of carbon dioxide*

\begin{tabular}{|c|c|c|c|c|c|c|c|c|c|c|c|}
\hline \multirow[b]{2}{*}{ Period } & \multirow[b]{2}{*}{ Dog } & \multirow[b]{2}{*}{ Days } & \multirow[b]{2}{*}{$\mathrm{Na}$} & \multirow[b]{2}{*}{$\mathrm{Cl}$} & \multirow{2}{*}{$\mathrm{Cl}-\frac{\mathrm{Na}}{1.3}$} & \multirow[b]{2}{*}{$\mathbf{K}$} & \multirow[b]{2}{*}{ Knt } & \multirow[b]{2}{*}{$\mathbf{N}$} & \multicolumn{3}{|c|}{ Intracellular shifts } \\
\hline & & & & & & & & & $\mathrm{Na}$ & $\mathbf{K}$ & Knt \\
\hline \multirow[t]{2}{*}{$7 \% \mathrm{CO}_{2}$} & $\begin{array}{l}\mathrm{G} \\
\mathrm{H} \\
925 \\
926 \\
929 \\
932 \\
942 \\
944\end{array}$ & $\begin{array}{l}7 \\
7 \\
7 \\
7 \\
7 \\
7 \\
7 \\
7\end{array}$ & $\begin{array}{l}m E q \\
-122 \\
-23 \\
-76 \\
=26 \\
=54 \\
=22 \\
+17 \\
-18\end{array}$ & $\begin{array}{l}m E q \\
-120 \\
+\quad 1 \\
-\quad 87 \\
-18 \\
-84 \\
-21 \\
-24 \\
-66\end{array}$ & $\begin{array}{l}m E_{q} \\
-26 \\
+19 \\
-29 \\
+2 \\
-42 \\
-4 \\
-37 \\
-52\end{array}$ & $\begin{array}{r}m E_{q} \\
+12 \\
+8 \\
-30 \\
-4 \\
-19 \\
-22 \\
0 \\
-12\end{array}$ & $\begin{array}{l}m E q \\
+40 \\
+54 \\
+16 \\
-12 \\
-21 \\
-25 \\
-11 \\
-10\end{array}$ & $\begin{array}{c}g \\
-10.3 \\
-17.1 \\
-16.9 \\
+\quad 3.1 \\
+\quad 0.9 \\
+1.2 \\
+4.0 \\
+0.9\end{array}$ & $\begin{array}{l}m E q \\
-20 \\
-87 \\
-5 \\
-39 \\
+13 \\
-12 \\
+22 \\
+40\end{array}$ & $\begin{array}{l}m E q \\
+15 \\
+7 \\
-28 \\
-5 \\
-16 \\
-22 \\
0 \\
-11\end{array}$ & $\begin{array}{l}m E q \\
+44 \\
+38 \\
+20 \\
-11 \\
-17 \\
-24 \\
-10 \\
-14\end{array}$ \\
\hline & \multicolumn{2}{|c|}{ Average } & -41 & -52 & -21 & -8 & +4 & -4.5 & -11 & -8 & +3 \\
\hline \multirow[t]{2}{*}{$11 \% \mathrm{CO}_{2}$} & $\begin{array}{c}\mathrm{G} \\
\mathrm{H} \\
925 \\
926 \\
929 \\
932 \\
942 \\
944\end{array}$ & $\begin{array}{l}9 \\
9 \\
9 \\
9 \\
7 \\
7 \\
8 \\
8\end{array}$ & $\begin{array}{r}-97 \\
-93 \\
-105 \\
+\quad 14 \\
-40 \\
-26 \\
+68 \\
-\quad 7\end{array}$ & $\begin{array}{l}-124 \\
-137 \\
-120 \\
-\quad 1 \\
-76 \\
-\quad 25 \\
+15 \\
-75\end{array}$ & $\begin{array}{l}-49 \\
-65 \\
-39 \\
-12 \\
-45 \\
-5 \\
-37 \\
-70\end{array}$ & $\begin{array}{l}-17 \\
-48 \\
-47 \\
-7 \\
-26 \\
-24 \\
+28 \\
-19\end{array}$ & $\begin{array}{l}+17 \\
+51 \\
+34 \\
+3 \\
+6 \\
-34 \\
+24 \\
+9\end{array}$ & $\begin{array}{r}-12.5 \\
-36.6 \\
-30.0 \\
-3.6 \\
-7.4 \\
+3.6 \\
+1.4 \\
-10.4\end{array}$ & $\begin{array}{l}+52 \\
+51 \\
+28 \\
-62 \\
+15 \\
-64 \\
+11 \\
+54\end{array}$ & $\begin{array}{l}-12 \\
-45 \\
-44 \\
-8 \\
-26 \\
-23 \\
+27 \\
-16\end{array}$ & $\begin{array}{l}+24 \\
+54 \\
+36 \\
+4 \\
-3 \\
-34 \\
+25 \\
+7\end{array}$ \\
\hline & \multicolumn{2}{|c|}{ Average } & -36 & -68 & -40 & -20 & +12 & -11.9 & +11 & -18 & +14 \\
\hline \multirow[t]{2}{*}{$17 \% \mathrm{CO}_{2}$} & $\begin{array}{l}926 \\
929 \\
932 \\
942 \\
944\end{array}$ & $\begin{array}{r}6 \\
6 \\
11 \\
9 \\
7\end{array}$ & $\begin{array}{r}+124 \\
+69 \\
-\quad 65 \\
-\quad 9 \\
+\quad 7\end{array}$ & $\begin{array}{l}+132 \\
+57 \\
-83 \\
-91 \\
-46\end{array}$ & $\begin{array}{l}+37 \\
+4 \\
-33 \\
-84 \\
-51\end{array}$ & $\begin{array}{l}-3 \\
-59 \\
-29 \\
-53 \\
-46\end{array}$ & $\begin{array}{l}+63 \\
-23 \\
+44 \\
-13 \\
+2\end{array}$ & $\begin{array}{l}-24.6 \\
-13.4 \\
-27.0 \\
-14.7 \\
-17.9\end{array}$ & $\begin{array}{l}-92 \\
-27 \\
+10 \\
+68 \\
+41\end{array}$ & $\begin{array}{l}-12 \\
-61 \\
-28 \\
-50 \\
-45\end{array}$ & $\begin{array}{l}+56 \\
-11 \\
+45 \\
-8 \\
-2\end{array}$ \\
\hline & \multicolumn{2}{|c|}{ Average } & +25 & -6 & -25 & -38 & +15 & -19.5 & 0 & -39 & +16 \\
\hline
\end{tabular}

* Data for each period have been accumulated separately.

$\dagger \mathrm{Kn}=\mathrm{K}$ corrected for $\mathrm{N}$.

3) Organic acids, sulfate, and body weight. Organic acid excretion was measured in one dog and was found to be augmented at each level of carbon dioxide exposure. A similar pattern for sulfate excretion (measured in one dog) was also observed. More extensive but similar observations of organic acid and sulfate excretion have been described previously during exposure to $12 \%$ carbon dioxide (5). Eight of the ten animals lost less than $1 \mathrm{~kg}$ in body weight (average, $0.4 \mathrm{~kg}$ ), and two animals (944 and D) lost 1.8 and $2.1 \mathrm{~kg}$, respectively.

\section{Discussion}

The present study has defined the chronic steady-state adjustments of acid-base equilibrium in dogs exposed to a wide range of carbon dioxide tensions. These observations, which have been made during prolonged uninterrupted exposure within an environmental chamber, serve to characterize descriptively the integrated re- sponse of kidneys and body buffers to graded degrees of chronic hypercapnia.

As shown in Figure 3, the increment in plasma bicarbonate concentration that occurred at each level of chronic hypercapnia was more than double that observed previously during comparable degrees of acute hypercapnia (2). At a carbon dioxide tension of $110 \mathrm{~mm} \mathrm{Hg}$, for example, the rise in bicarbonate concentration during the chronic steady state was more than $20 \mathrm{mEq}$ per $\mathrm{L}$ as compared to the 7 to $8 \mathrm{mEq}$ per $L$ observed during acute studies (2). The greater effectiveness of the chronic adaptive mechanisms, in terms of defense of $\mathrm{pH}$, is clearly illustrated by Figure 4, which demonstrates that hydrogen ion concentration increases by only 0.32 nmoles per $\mathrm{L}$ per $\mathrm{mm}$ of $\mathrm{Pco}_{2}$ rather than by 0.77 nmoles per $L$ as in acute hypercapnia (2).

A matter of perhaps even greater physiologic interest is that, just as during acute hypercapnia, the relationship between hydrogen ion concentration 
and $\mathrm{PCO}_{2}$ is remarkably linear. The factors responsible for the observed linear hydrogen ion/ $\mathrm{PCO}_{2}$ relationship are not clear, but it is tempting to speculate that bicarbonate generation by the kidneys may be regulated by a feedback process sensitive to extracellular hydrogen ion concentration. On the other hand, the possibility must be considered that primary changes in intracellular acidity control renal generation of bicarbonate and that such changes are responsible for the linear regression of extracellular hydrogen on $\mathrm{PCO}_{2}$. Whatever control mechanisms are operating in chronic hypercapnia, they would appear to be "satisfied" with a bicarbonate concentration that falls significantly short of returning extracellular $\mathrm{pH}$ to normal levels.

Regardless of the processes that produce the steady-state adjustments of acid-base parameters, the quantitative results of these adjustments are remarkably uniform. The narrow limits of the physiologic response of the fully adapted organism to varying degrees of chronic hypercapnia are indicated by the significance bands calculated from these data (Figure 5 and Table II). At any elevation of $\mathrm{PCO}_{2}$ up to $100 \mathrm{~mm} \mathrm{Hg}$, plasma hydrogen ion and bicarbonate concentrations would be expected to fall within zones no wider than 9 nmoles per $\mathrm{L}$ and $6 \mathrm{mEq}$ per $\mathrm{L}$, respectively. The reproducible nature of the carbon dioxide response curves, as evidenced by such significance bands, should provide a useful reference point from which to investigate further the regulation of renal bicarbonate generation and reabsorption during chronic hypercapnia; variations in both acid excretion and bicarbonate conservation during potassium depletion, hypoxia, and other abnormal states may well throw light on the intimate renal compensatory mechanisms that defend $\mathrm{pH}$ during the hypercapneic state.

The data on acid excretion appear, in a general way, to provide a satisfactory explanation for the changes that are seen in plasma acid-base composition. During each period of exposure net acid excretion rose to levels well above control, the increment being larger with each successive level of hypercapnia. This increment was, in fact, far in excess of that necessary to account for the rise in extracellular bicarbonate concentration and even in excess of the amount necessary to account for an equivalent rise in intracellular alkali stores. It seems likely that part of the "excess" acid excreted in the urine simply represents an increase in endogenous acid load, as reflected by the rise in both organic acid and sulfate excretions noted at each level of hypercapnia.

It is apparent that data for normal man, analogous to those presented here for the dog, would be of considerable physiologic interest. Knowledge of the "normal" response to a given degree of chronic hypercapnia would also have potential clinical value, since it would provide the most satisfactory basis for revealing the presence of complicating metabolic acid-base disturbances in the patient with pulmonary insufficiency. Recent observations in our laboratory indicate, however, that physiologic studies of prolonged hypercapnia in man are not feasible because carbon dioxide concentrations of even 5 or $6 \%$ are not tolerated for more than a few hours. Since complicating metabolic disturbances appear to occur with considerable frequency in patients with pulmonary disease (7), it seems reasonable to ask whether there is sufficient evidence to justify extrapolation of the present data to the patient with carbon dioxide retention. It is well known that man and dog respond in a closely similar fashion to a variety of metabolic acid-base disorders. In addition both species have recently been shown to behave in a nearly identical fashion in response to acute respiratory acidosis (3). Finally, it is apparent from the significance bands for the dog that at a $\mathrm{PCO}_{2}$ of 40 to $45 \mathrm{~mm}$ of $\mathrm{Hg}$ the values of $\mathrm{pH}$ and bicarbonate are virtually identical to those seen in normocapneic man. This latter observation indicates that the normal hydrogen ion $/ \mathrm{PCO}_{2}$ point for man falls on the line that is the locus of the chronic steady-state hydrogen ion $/ \mathrm{PCO}_{2}$ points for the dog. All of these above considerations suggest that the response of man and dog to chronic hypercapnia should be closely similar. If this interpretation is correct, the significance bands reported here should provide a rational basis for both identification and management of metabolic acid-base disturbances in the patients with chronic pulmonary insufficiency. Observations in patients with chronic respiratory acidosis who appear to be free from complicating metabolic acid-base disturbances (due to diuretics, vomiting, etc.) should provide 
further information concerning the clinical applicability of the present experimental findings.

\section{Summary}

Studies have been carried out in ten normal dogs in order to characterize the response of acidbase equilibrium to chronic, stepwise increases in arterial carbon dioxide tension. At each concentration of carbon dioxide $(7,11,17 \%)$ the animals were exposed for a period of at least 5 days to allow extracellular composition to reach a new steady state. The data demonstrate that every increment of $\mathrm{PCO}_{2}$ induced a rise in renal acid excretion and a marked rise in plasma bicarbonate concentration, but at no level of carbon dioxide tensions was the $\mathrm{pH}$ of the extracellular fluid restored to normal.

A notable finding was the strikingly linear relationship between hydrogen ion concentration and $\mathrm{PCO}_{2}$, each millimeter of increment in carbon dioxide tension inducing an average rise of 0.32 nmoles per $\mathrm{L}$ of hydrogen ion concentration. On the basis of this finding it has been suggested that a feedback mechanism sensitive to hydrogen ion concentration may govern the addition of bicarbonate to the extracellular fluid by the kidneys during chronic hypercapnia.

The response curves for plasma hydrogen ion and bicarbonate concentrations, when expressed in terms of $95 \%$ significance bands, indicate that a relatively narrow range of concentrations defines the anticipated physiologic response to a given degree of chronic hypercapnia. The possible application of such significance bands in delineating metabolic acid-base disturbances in chronic respiratory acidosis has been considered.

\section{Statistical Appendix}

To analyze the relationship between $\mathrm{PcO}_{2}$ and hydrogen ion concentration, it is first necessary to examine the variance in hydrogen ion concentration for homogeneity. The variances were found to be homogeneous when examined between animals at each level of carbon dioxide (control, 7, 11, and 17\%). The variances were pooled and were also found to be homogeneous between periods. A combined estimate of this variability, based on 61 degrees of freedom, was then obtained that represents an estimate of the experimental (biological and measurement) error. Standard linear regressions were then, calculated for each study. In all cases the correlation coefficients associated with these lines were greater than 0.92 .
Having established the essential linearity of the $\mathrm{H}^{+} /$ $\mathrm{PCO}_{2}$ relationship, we devised a method to determine whether single future observations of hydrogen ion concentration could be accounted for solely as a result of the response to uncomplicated chronic hypercapnia. If we assume a population of linear $\mathrm{H}^{+} / \mathrm{PcO}_{2}$ relationships, the mean population response can be estimated by averaging the slopes and intercepts of the observed regression lines. The variability of the slopes and intercepts of this population was also estimated from the data at hand. This allowed the computation of a standard error for the difference between a newly observed hydrogen ion value and the estimated mean population response at the same $\mathrm{PCO}_{2}$. The significance of the difference, assessed by computing the corresponding $t$ value, provides a test of the hypothesis that this new hydrogen ion value is the result of uncomplicated hypercapnia. This $t$ test may be conveniently performed at the $5 \%$ level by plotting the observed $\mathrm{H}^{+} / \mathrm{PCO}_{2}$ point on the chart containing the significance band. For a point falling outside the band, the associated hydrogen ion concentration is significantly different from the estimated mean response $(p<0.05)$.

\section{Acknowledgments}

The authors wish to thank Dr. Robert Berk for his valuable assistance in the statistical analysis of the data and Dr. William G. Cochran for his critique of the statistical methods employed.

\section{References}

1. Giebisch, G., L. Berger, and R. F. Pitts. The extrarenal response to acute acid-base disturbances of respiratory origin. J. clin. Invest. 1955, 34, 231.

2. Cohen, J. J., N. C. Brackett, Jr., and W. B. Schwartz. The nature of the carbon dioxide titration curve in the normal dog. J. clin. Invest. 1964, 43, 777.

3. Brackett, N. C., Jr., J. J. Cohen, and W. B. Schwartz. The carbon dioxide titration curve of normal man; the effect of increasing degrees of acute hypercapnia on acid-base equilibrium. New Engl. J. Med. 1965, 272, 6.

4. Schwartz, W. B., and L. Silverman. A large environmental chamber for the study of hypercapnia and hypoxia. J. appl. Physiol., in press.

5. Polak, A., G. D. Haynie, R. M. Hays, and W. B. Schwartz. Effects of chronic hypercapnia on electrolyte and acid-base equilibrium. I. Adaptation. J. clin. Invest. 1961, 40, 1223.

6. Van Ypersele de Strihou, C., P. F. Gulyassy, and W. B. Schwartz. Effects of chronic hypercapnia on electrolyte and acid-base equilibrium. III. Characteristics of the adaptive and recovery process as evaluated by provision of alkali. J. clin. Invest. 1962, 41, 2246.

7. Robin, E. D. Abnormalities of acid-base regulation in chronic pulmonary disease, with special reference to hypercapnia and extracellular alkalosis. New Engl. J. Med. 1963, 268, 917. 